\title{
VERSITA
}

DOI: 10.2478/aucft-2013-0001

\section{ESTIMATING WINE YEAST CONCENTRATION BY SPECKLE SIZE MEASUREMENT}

- research paper -

\author{
Dan CHICEA ${ }^{1}$, Ovidiu TIȚA ${ }^{2,3}$, Mihaela TIȚA $^{3}$, Ecaterina LENGYEL $^{3}$ \\ 1 Department of Environmental Sciences, University Lucian Blaga, Dr. Ion \\ Ratiu str., no 5-7, Sibiu, 550012, ROMANIA \\ 2 Pediatric Respiratory Medicine Research Center (CCMRP), Str. Pompeiu \\ Onofreiu Nr. 2 - 4, Sibiu, ROMANIA \\ 3 Faculty of Agricultural Sciences, Food Industry and Environmental \\ Protection, University Lucian Blaga, Dr. Ion Ratiu str., no 7-9, Sibiu, \\ 550012, ROMANIA
}

\begin{abstract}
A coherent light scattering experiment on aqueous suspension of Saccharomyces cerevisia yeast with a concentration that covers five orders of magnitude was performed. The average scattered light far field was recorded using a CCD. A computer code for image processing was written for this purpose and used for providing the first order statistics of the far interference field. The variation of the average speckle size with the particle concentration was analyzed and a very fast procedure for monitoring the yeast concentration is suggested. The possibility of monitoring the fermentation process by a fast assessment of the yeast concentration is suggested, as well.
\end{abstract}

Keywords: Coherent Light Scattering, Suspension, Particle Concentration, Sacharomyces cerevisiae ssp. ellipsoideus.

\footnotetext{
${ }^{1}$ Corresponding author. Mailing address: Department of Environmental Sciences, University Lucian Blaga, Dr. Ion Ratiu str., no 5-7, Sibiu, 550012, ROMANIA. E-Mail: dan.chicea@ulbsibiu.ro
}

Vol. XVII (2013), no.1 


\section{INTRODUCTION}

Yeasts are a eukaryotic microorganisms classified in the kingdom Fungi. Approximately 1500 species of yeasts have been described (Kurtzman and Fell, 2006). Most of the yeast species reproduce asexually by budding, although in a few cases by binary fission (Kurtzman and Fell, 2006). Yeasts are unicellular, although some species with yeast forms may become multicellular through the formation of a string of connected budding cells known as pseudohyphae, or true hyphae as seen in most moulds. Yeasts are classified into two groups, ascomycetous and basidiomycetous yeasts (Kreger-van Rij , 1994). For these two groups the asexual reproduction is different from one to the other. Anamorphic ascomycetous yeasts stretch all wall layers during asexual reproduction, process that is called holoblastic budding, while anamorphic basidiomycetous yeasts grow out the inner wall layer, thus producing an enteroblastic bud with a collarette (Kreger-van Rij and Veenhuis, 1971).

Ascomycetes yeasts are classified into the Saccharomycetes and Schizosaccharomycetes, while the basidiomycetes yeasts are distributed into Hymenomycetes, Urediniomycetes and Ustilaginomycetes (Kurtzman and Fell, 2006). Yeasts occupy different habitats, as aquatic, atmospheric, plant and soil. Consequently, they play various ecological and biotechnological roles such as bioremediation of toxic compounds (Kwon et al., 2002), fermentation of beverages (Lodolo et al., 2008) and food processing (Viljoen et al., 2003).

Nowadays, comprehensive research is being done with respect to the methods of obtaining lipids and phospholipids from lipid biocomponents, in order to identify new methods for obtaining liposomal substances, needed by the pharmaceutical, cosmetic and medical industry. At present, egg lecithin is commonly used instead. The use of this source has several drawbacks though; one of them laying in the fact that it oxidizes easily. Eukaryotes (yeasts, fungi, algae) are the main microorganisms that produce lipids and phospholipids. Of great interest to microbiology and biotechnology are the researches conducted in the field of phospholipids synthesis, of obtaining phospholipids from microorganisms and of optimizing culture media for their cultivation. Monitoring yeast concentration is therefore of interest during a biotechnological process.

The work described here presents an unconventional method based on coherent light scattered on a suspension and on analyzing the scattered far

Vol. XVII (2013), no.1 
field that might be used in assessing in a fast manner the variation of the concentration of the yeast in aqueous suspension, as fermentation of beverages is.

\section{MATERIALS AND METHODS}

\section{a) Sample preparation}

The wine yeast that was used in this study is Saccharomyces cerevisiae ssp. ellipsoideus. The yeast was in dry state. Initially $0.2 \mathrm{~g}$ of dry yeast was dissolved in $20 \mathrm{ml}$ of deionised water and allowed to become moisturized for 24 hours. This initial sample had a concentration of $10 \mathrm{~g} / \mathrm{l}$, expressed in grams of dry yeast per litre and the concentrations mentioned in this work are consistent with in this respect. From this initial diluted sample $4 \mathrm{ml}$ were extracted and placed in a transparent glass cuvette having a square section of $1 \times 1 \mathrm{~cm}^{2}$ and was used as a target for the light scattering experiment. The other samples were obtained by successive dilution. 29 samples were used in the study reported here and the concentration range was $\left[7.6 \cdot 10^{-03}-10\right] \mathrm{g} / \mathrm{l}$.

\section{b) Light scattering experimental setup.}

The schematic of the experiment is presented in Figure 1. The coherent light source was a laser diode that had a wavelength of $633 \mathrm{~nm}$ and a constant power of $20 \mathrm{~mW}$. No polarizer was placed between the laser and the glass cuvette, which had the optical pass equal to $1 \mathrm{~cm}$; D was $0.162 \mathrm{~m}$. The camera was a Philips SPC900NC CCD with the optical system removed. The center of the CCD array was placed $0.007 \mathrm{~mm}$ apart from the beam making the measurement angle to be $2^{\circ} 28^{\prime} 27.1^{\prime}$ '.

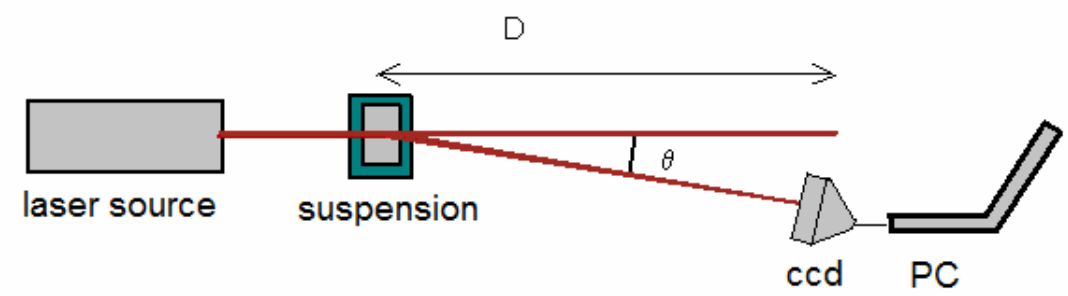

Figure 1. A view from above of the schematic of the experiment.

Vol. XVII (2013), no.1 
As the optical system of the camera was removed, the recorded images are the direct interference of the scattered wavelets on the CCD detection array. The resolution was $640 \times 480$ pixels, the frame rate was 10 frames per second (fps) and the color depth was 16 bits. A frame taken from the movie having a very small concentration, that is $1.7 \cdot 10^{-1} \mathrm{~g} / \mathrm{l}$, is presented in Figure 2. Examining Figure 2 we notice the speckle aspect, given by a succession of maxima and minima randomly distributed across the far interference field.

Each of the 29 samples was prepared by successive dilution, as previously described and became target for the laser beam. A movie lasting for 30 seconds was recorded for each sample and processed later on.

\section{c) Data processing procedure}

The movie was processed with a computer program written for this purpose. The program reads the movie, extracts frame by frame in a $640 \times 480$ array of intensity levels. We can note $\mathrm{I}(\mathrm{i}, \mathrm{j})=\mathrm{I}(\mathrm{xi}, \mathrm{yj})$ the intensity recorded by the cell $(i, j)$ of the $C C D$, hence by the pixel $(i, j)$ of the array of pixels the image consists of.

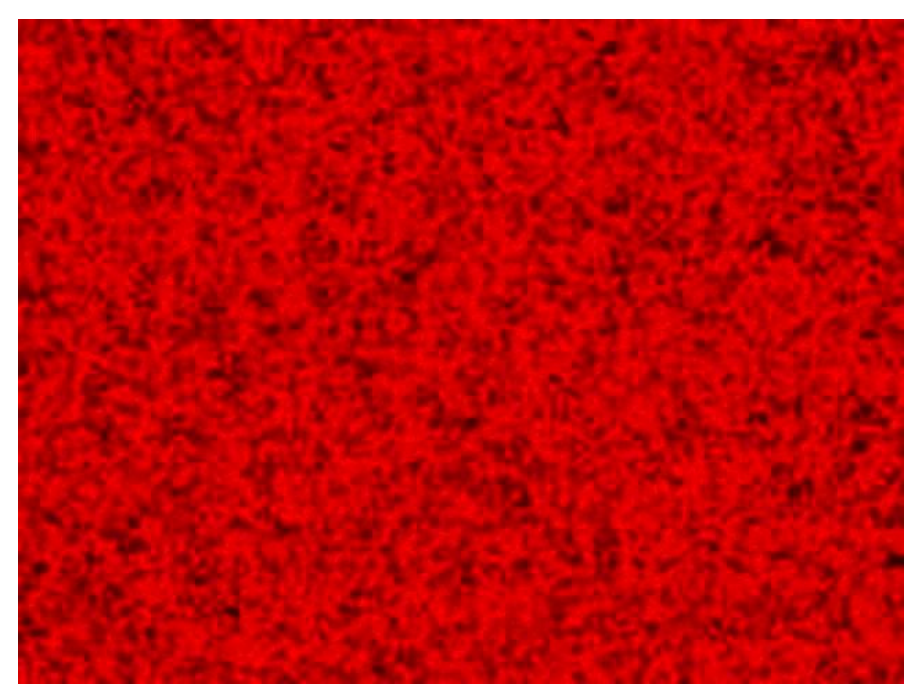

Figure 2. A frame taken from the movie recorded for the sample having a clay concentration of $1.7 \cdot 10^{-1} \mathrm{~g} / \mathrm{l}$.

Vol. XVII (2013), no.1 
In (Goodman, 1984), (Chicea, 2010), (Chicea, 2012) and (Piederriere et al., 2005) the average speckle size is calculated as the normalized autocovariance function of the intensity speckle pattern acquired in the observation plane:

$$
c_{l}(\Delta x, \Delta y)=\frac{\left.F T^{-1}[F T[I(x, y))]^{2}\right]-\langle I(x, y)\rangle^{2}}{\left\langle I(x, y)^{2}\right\rangle-\langle I(x, y)\rangle^{2}}
$$

where FT is the Fourier transform, $<>$ stands for spatial average, $\operatorname{cl}(\Delta \mathrm{x}, 0)$ is a horizontal and $\mathrm{cl}(0, \Delta \mathrm{y})$ a vertical profile. The $\mathrm{cl}(\Delta \mathrm{y}, \Delta \mathrm{y})$ function defined in (1) has a 0 base and the width of the function provides a reasonable measure of the speckle size, as stated in (Piederriere et al., 2005).

In this paper a different approach is proposed to calculate the speckle size. For each vertical profile of a frame the normalized autocorrelation function (Bracewell, 1999) is calculated. The autocorrelation function for each sample was calculated as:

$$
A_{n}(s)=\frac{\langle I(n, j) * I(n, j+s)\rangle}{\langle I(n, j) * I(n, j)\rangle}
$$

where the angle brackets denote averages over the coordinate $\mathrm{y}, \mathrm{n}$ represents the number of the vertical profile and $\mathrm{s}$ is the autocorrelation distance.

The speckle size for $n$-th profile, (the profile speckle size) is defined as the value of the s (pixel number or distance, if multiplied by the pixel size on the CCD) where the autocorrelation function decreases to 1/e. An average of the profile speckle sizes is calculated for each image; therefore we can define this average as the average speckle size for that particular image. A vertical profile is presented in Figure 3.

The procedure that was used in this work is apart of the procedures described in (Piederriere et al., 2004), (Piederriere et al., 2004a). This algorithm has been tested on simulated profiles with absolute value of sinus type peaks having different half periods added and the average speckle size calculated as described above provides a reasonably accurate measure of the speckle size. More details on using the algorithm, on testing it and on the results of calculating the speckle size using it are presented in detail in (Chicea, 2007). The speckle size was extracted from each frame of the movie and an average was computed for that movie, hence for that sample. The standard deviation was computed for the collection of data representing the speckle size calculated for each frame of the movie and was considered to be the error in assessing the average speckle size for that particular sample. Consequently it represents the size of the error bar of the data plotted in Figure 4.

Acta Universitatis Cibiniensis Series E: FOOD TECHNOLOGY

Vol. XVII (2013), no.1 


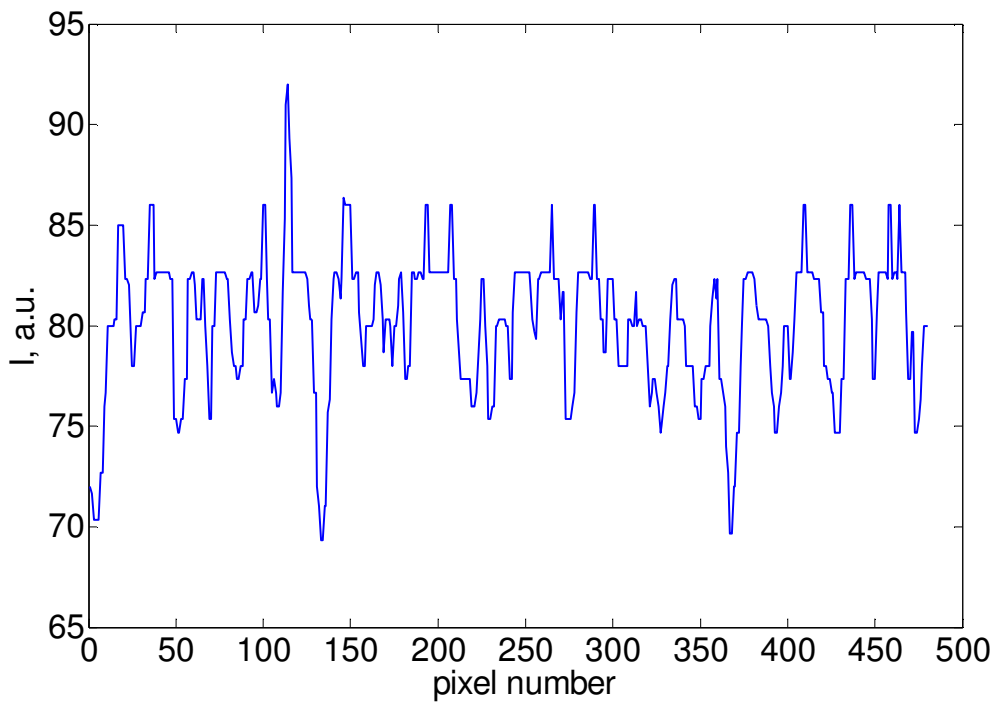

Figure 3. A vertical profile extracted from the movie recorded for the sample having an yeast concentration of $1.7 \cdot 10^{-1} \mathrm{~g} / 1$.

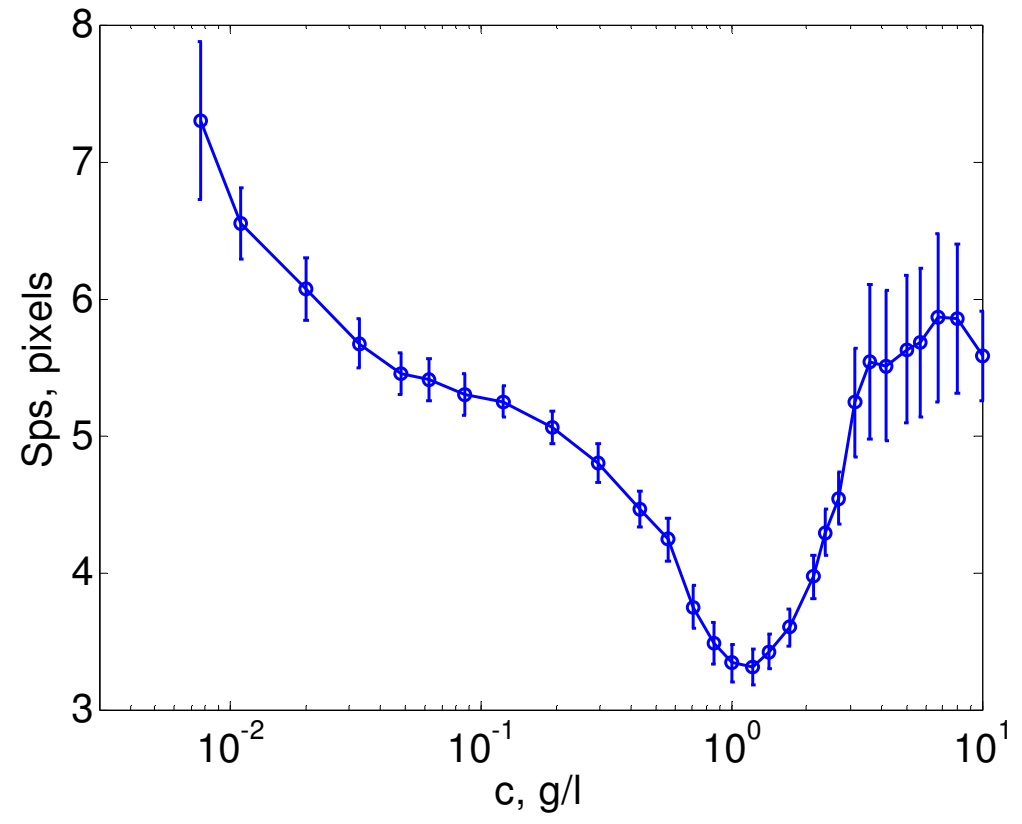

Figure 4. The speckle size, in pixels, versus yeast concentration; $\mathrm{X}$ axis is logarithmic.

Vol. XVII (2013), no.1 


\section{RESULTS AND DISCUSSIONS}

The samples were prepared using the procedure described in the previous section starting with a concentration of $10 \mathrm{~g} / \mathrm{l}$ and decreasing it by successive dilution down to $7.59 \cdot 10^{-3} \mathrm{~g} / \mathrm{l}$. The speckle size was computed for each sample and the results are presented in Figure 4, which has a logarithmic concentration axis, as the concentration values stretch over five orders of magnitude. The error bars on each data point represent the standard deviation computed on the collection of 300 values of the speckle size for each of the 300 frames recorded for each sample, as previously described.

Examining Figure 4 we notice that the average speckle size presents a clear decreasing trend in the very small concentration range, that is smaller than 10 $\mathrm{g} / \mathrm{l}$, that fast increase over the concentration range, from $1 \mathrm{~g} / \mathrm{l}$ to $4 \mathrm{~g} / \mathrm{l}$, followed by a small increase and a plateau for bigger concentrations. The decrease is similar with the results reported in (Piederriere et al., 2004a). The increase presented in Figure 4 is different from the results reported (Piederriere et al., 2004) and (Chicea, 2007) where the samples were different and the concentration was expressed as optical depth. The difference can be explained by the concentration range used in this work, which covers a wider range than in (Piederriere et al., 2004), deeper in the multiple scattering range, as described above. If a smaller range is extracted from the plot in Figure 4, a linear variation will appear.

Similar work is described in (Chicea, 2013), where clay particles were the scattering centers. The results in (Chicea, 2013) reveal an uncertain behavior in the very small concentration range, followed by a decrease and than by an increase of the speckle size with the concentration. The differences can be explained by the range of the optical depth of the sample (Chicea, 2007).

This experimental procedure that has Figure 4 as output, suggests a simple ansatz that can be used in monitoring the fermentation process in aqueous environment. As the fermentation carries on, the yeast concentration increases up to a maximum than it decreases as the nutrients were consumed. If a small amount of the aqueous fermentation environment is extracted from the reactor on a regular time basis and is analyzed following the procedure described in this work, a curve representing the time variation of the speckle size can be plot. As the fermentation carries on, an initial decrease of the speckle size is supposed to appear. It should be followed by an increase of the speckle size, produced by the continuous increase of the yeast concentration, accordingly to the increasing part of the curve in Figure 4 and

Vol. XVII (2013), no.1 
than a plateau in the speckle size. As time passes and the fermentation process intensity decreases, the yeast concentration decreases and the speckle size is supposed to follow the same trend but in reverted manner. A decrease of the speckle size is supposed to occur, followed by an increase as the yeast concentration decreases further on. Plotting such a curve, speckle size versus time, derived using the procedure described in this work, might enable monitoring the fermentation process by monitoring the yeas concentration. This procedure requires experimental verification though, as other particles having a size of the order of microns might lead to false results.

\section{CONCLUSIONS}

The work described in this article states that a simple physical procedure using the far interference field of a coherent light beam incident on a suspension can be used to assess the yeast concentration in water. The far interference field is recorded using a CCD and the movie is processed later on using a program written for this purpose that uses an algorithm previously tested (Chicea, 2007), (Chicea, 2013). The average speckle size variation with the concentration was found to be monotone (decreasing with the concentration) over an extended concentration range, up to $1 \mathrm{~g} / \mathrm{l}$. For bigger concentrations there is a fast increase in the range [1 - 4] g/l followed by a plateau. This behaviour suggests a simple yet very fast procedure in monitoring the fermentation by estimating the yeast concentration variation in time.

This suggested procedure must be verified experimentally prior of using it for commercial applications, as the presence of other micron sized particles in suspension might lead to modified results.

\section{REFERENCES}

1. Bracewell R., The Autocorrelation Function, The Fourier Transform and Its Applications, 3rd ed. New York: McGraw-Hill, 1999, 40-45

2. Chicea D., A Study of Nanoparticle Aggregation by Coherent Light Scattering, Current Nanoscience, 2012, 8(6), 259-265.

3. Chicea D., Estimating Particle Concentration in Natural Water by Speckle Size Measurement, 11th WSEAS International Conference on Environment, Ecosystems and Development (EED '13), Brasov, Romania, June 1-3, 2013, accepted.

Vol. XVII (2013), no.1 
4. Chicea D., Nanoparticles and Nanoparticle Aggregates Sizing by DLS and AFM, Journal of Optoelectronics and Advanced Materials vol. 4, issue 9, 2010, pp.1310-1315.

5. Chicea D., Speckle Size, Intensity and Contrast Measurement Application in Micron-Size Particle Concentration Assessment, European Physical Journal Applied Physics 2007, 40, 305-310, doi: 10.1051/epjap:2007163.

6. Goodman J.W., Statistical Properties of Laser Speckle Patterns, in Laser speckle and related phenomena, Vol.9 in series Topics in Applied Physics, J.C. Dainty, Ed., Springer-Verlag, Berlin, Heidelberg, New York, Tokyo, 1984.

7. Kreger-van Rij N. J. W. General classification of the yeasts. In The yeasts: a Taxonomic Study. 3rd edition. Edited by N. J. W. Kreger-van Rij, Elsevier, 1-44, Amsterdam, 1984.

8. Kreger-van Rij, N. J. W., Veenhuis, M. A comparative study of the cell wall structure of basidiomycetous and related yeasts. Journal of General Microbiology, 1971, 68, 87-95.

9. Kurtzman C.P., Fell J.W., Yeast Systematics and PhylogenyImplications of Molecular Identification Methods for Studies in Ecology, in Biodiversity and Ecophysiology of Yeasts, The Yeast Handbook, Edited by C. Rosa \& G. Péter, Springer-Verlag, 11-30, 2006.

10. Kwon H. K., Woo S. H., Park J. M., Degradation of tetracyanonickelate (II) by Cryptococcus humicolus MCN2. FEMS Microbiology Letters, 2002, 214, 211-216.

11. Lodolo E. J., Kock J. L. F., Axcell B. C., Brooks M. The yeast Saccharomyces cerevisiae: the main character in beer brewing. FEMS Yeast Research, 2008, 8, 1018-1036.

12. Piederriere Y., Boulvert F., Cariou J., Le Jeune B., Guern Y., Le Brun G., Backscattered speckle size as a function of polarization: influence of particle-size and concentration, Optics Express, 2005, 13, 5030 5039.

13. Piederrière Y., Cariou J., Guern Y., Le Jeune B., Le Brun G., Lotrian J., Scattering through fluids: speckle size measurement and Monte Carlo simulations close to and into the multiple scattering, Optics Express 2004, 12, 176-188.

14. Piederrière Y., Le Meur J., Cariou J., Abgrall J.F., Blouch M.T., Particle aggregation monitoring by speckle size measurement;

Vol. XVII (2013), no.1 
application to blood platelets aggregation, Optics Express 2004a, 12, $4596-4601$.

15. Viljoen B. C., Knox A. M., de Jager P. H., Lourens-Hattingh A. Development of yeast populations during processing and ripening of blue veined cheese. Food Technology and Biotechnology, 2003, 41: 291-297. 